CORRECTION

\title{
Correction: Optimizing psychedelic compounds for neuropsychiatric therapy
}

Scott M. Thompson (iD)

Neuropsychopharmacology (2021) 46:1545; https://doi.org/10.1038/s41386-021-01021-6

Correction to: Neuropsychopharmacology https://doi.org/10.1038/ s41386-021-01004-7, published online 05 April 2021

The original version of this article unfortunately mischaracterized the pharmacology of ibogaine.

The corrected text is as follows:

The recent report in Nature from Cameron et al. (2021) ${ }^{4}$ offers tantalizing evidence that non-hallucinogenic psychedelic compounds may possess beneficial actions. The authors started with ibogaine, a powerful hallucinogenic plant alkaloid with low affinity for serotonin and dopamine transporters, as well as kappa opioid receptors ${ }^{4,5}$. They then synthesized a novel analog of ibogaine called tabernanthalog (TBG). TBG is shown to have unique pharmacological properties and to lack many of the negative side effects of ibogaine. 